\title{
Objektive Informationsquelle, Watchdog und Sprachrohr der Bürger? Die Bewertung der gesellschaftlichen Leistungen von Medien durch die Bevölkerung
}

\author{
Nayla Fawzi
}

Online publiziert: 3. April 2020

(C) Der/die Autor(en) 2020

Zusammenfassung Die Massenmedien sehen sich derzeit einer umfassenden öffentlichen Kritik ausgesetzt, die u.a. die politischen und sozialen Funktionen der Medien ins Visier nimmt: Von „Lügenpresse“-Vorwürfen über das Infragestellen der journalistischen Autonomie bis zu Kritik an der Breite des Meinungsspektrums in der Berichterstattung. Es stellt sich daher die Frage, inwiefern die Medien aus der Perspektive des Publikums in der Lage sind, die von ihnen erwarteten Leistungen tatsächlich zu erfüllen. Vor diesem Hintergrund liefert die Studie auf Basis einer repräsentativen Bevölkerungsumfrage deskriptive Befunde zur Zufriedenheit mit den Leistungen der Medien und untersucht, welche Faktoren diese Performanzbewertungen erklären. Dabei zeigt sich, dass die Medien aus Sicht der Bürger*innen ihre Kritik- und Kontrollfunktion sowie die Informations- und Meinungsbildungsfunktion relativ gut erfüllen. Deutlich weniger zufrieden ist das Publikum mit den Integrations-, Artikulations- und Orientierungsleistungen. Politisches Interesse, Demokratiezufriedenheit und Politikvertrauen sowie selektive Mediennutzung, HostileMedia-Wahrnehmung und Presumed media influence sind ausschlaggebend für diese Bewertungen; das Medienrepertoire spielt dagegen keine Rolle.

Schlüsselwörter Funktionen der Medien · Medienvertrauen · Lügenpresse · Medienleistungen $\cdot$ Medienperformanz $\cdot$ Medienqualität

Dr. N. Fawzi $(\bowtie)$

Institut für Kommunikationswissenschaft und Medienforschung, Ludwig-Maximilians-Universität München, Oettingenstr. 67, 80538 München, Deutschland

E-Mail: fawzi@ifkw.lmu.de 


\section{Objective information source, watchdog and citizens' mouthpiece? The mass media's performance from an audience perspective}

Abstract The mass media currently find themselves increasingly under public fire: From "lying press" accusations to questioning of the journalistic autonomy to fundamental media attacks by politicians. Many points of criticism address the normative functions that are expected from the mass media in democratic societies: information, public forum, opinion forming, watchdog, socialization, mobilization, orientation or social integration. These functions are the central theoretical basis for intensively studied concepts such as media quality or media trust. Quality criteria such as diversity or independence are derived from the normative media functions. Media trust is often defined as the belief that the media performance will be in line with the normative expectations, i.e. that the media will fulfil the functions ascribed to them. However, empirical studies e.g. on media quality do not allow direct conclusions on the fulfilment of normative functions, since the quality criteria of media content are examined at a lower level of abstraction and do not comprehensively cover the normative functions (e.g. mobilization).

What recipients expect from the media and how they evaluate them depends on their role that they adopt. In their role as consumers they are interested in maximizing individual benefits; the evaluation criteria here correspond to expected gratifications such as entertainment. In their role as citizens, recipients take a perspective of society as a whole. These expectations of the media can be derived from the demands that the various models of democratic theory impose on the political public sphere. From this citizens' perspective, the normative functions of journalistic media can thus be understood as services that they provide for society, the media's performance.

Against this background, this study analysis from a citizens' perspective to what extent the media are actually able to fulfil the expected performance and which individual political and media-related variables predict these evaluations. Based on a representative survey of the German population $(N=1005$, CATI), the descriptive results show, that the audience perceives the media to fulfill their watchdog, information and opinion formation function satisfactorily, while a larger part is dissatisfied with the media's fulfillment of their integration, articulation and orientation function. A more positive assessment is gained by a more general question, whether the media fulfil their democratic performance in general. Remarkably, this received by far the most support compared to the concrete functions: $61 \%$ of those surveyed are "all in all" satisfied with the performance of the media.

Which factors correlate with the evaluation of the media performance? Political interest, satisfaction with democracy, political confidence and satisfaction with the current economic situation are positively related to satisfaction with media performance. In contrast, media use does not play a role. Media performance evaluations are thus independent of whether the recipients tend to consume rather public broadcasting or tabloid media. As far as media use is concerned, selective exposure to congruent media content alone is decisive. With regard to attitudes towards the media, the following correlation can be seen: the less strongly respondents perceive the media to be hostile towards their own political attitudes, the better they evaluate media performance. There is also a positive correlation to the perceived media 
dependency and presumed media influence. Perceived information overload, on the other hand, does not play a role,

The results show that a significant part of citizens is disappointed with the performance of the media in terms of what journalism offers them. Future research should focus in more detail on the dissatisfied population groups. If a citizen has the feeling that the media do not inform objectively, if he/she does not find his/her views in any established media and thus does not feel represented by the media, or he/she convinced that the media are not able to criticize politicians and their daily decisions, this can have a negative impact on political participation and social cohesion. Particularly in times of growing populist forces attacking the liberal pillar of democracy and the media, the integrative and mediating performances of the media are needed, as is their independence from politics and business. This could be one reason for the increasing shift of this population group away from the established media towards alternative and partisan media that are more in line with their own political interests. Such developments bear the risk that these groups will leave the common public discourse, which will increase the polarization of society. The positive influence of satisfaction with democracy and trust in politics on media performance evaluations also shows that those who already feel neglected by politics transfer this impression to the media.

Keywords Normative media functions - Trust in media - Media performance Media quality $\cdot$ Lying press

\section{Einleitung}

Medienkritik und Qualitätsdebatten begleiten die traditionellen Massenmedien seit deren Bestehen (vgl. Arnold 2009; McQuail 1992). Entsprechend kann die Kommunikationswissenschaft einen umfangreichen Forschungsstand zur (wahrgenommenen) Medienqualität vorweisen (z. B. Arnold 2009; Neuberger 2014; Gehrau 2008; Wolling 2004). Die öffentliche Kritik an den Medien scheint seit einigen Jahren jedoch eine neue Dimension zu erreichen: von „Lügenpresse“-Vorwürfen über Beleidigungen und Angriffe gegen Journalist*innen bis zu medienfeindlichen Äußerungen von Politiker*innen. Folglich wird vielerorts eine Vertrauenskrise der Medien beschworen, auch wenn sich dieser Eindruck anhand von Längsschnittbefragungen nicht unbedingt belegen lässt (vgl. z. B. Reinemann et al. 2017; Ziegele et al. 2018). Die Debatte belebte in jedem Fall die deutschsprachige Forschung zum Medienvertrauen (vgl. Beiträge in Blöbaum 2016; Otto und Köhler 2018; Schultz et al. 2017; Fawzi und Obermaier 2019).

Für die beiden Konstrukte Medienvertrauen und Medienqualität sind die gesellschaftlichen Funktionen der Medien der zentrale theoretische Ausgangspunkt. Medienvertrauen wird häufig definiert als Überzeugung, dass die Leistungen der Medien mit den an sie gestellten Erwartungen übereinstimmen, dass die Medien die ihnen zugeschriebenen Funktionen also erfüllen werden (vgl. z. B. Jackob 2012; Müller 2013). Darüber hinaus werden zentrale Qualitätskriterien wie Vielfalt oder Unabhängigkeit von den normativen Medienfunktionen abgeleitet (vgl. Arnold 2009; 
Neuberger 2004; Schatz und Schulz 1992; Wellbrock und Klein 2014). Eine Analyse der Medienqualität auf niedrigerem Abstraktionsniveau hat den Vorteil, dass die Kriterien, die sich auf Medieninhalte beziehen (z. B. Vielfalt) einfacher empirisch überprüfbar sind. Die konkreten Folgen für die Gesellschaft, wie sie zum Beispiel in der Meinungsbildungsfunktion formuliert werden, bleiben allerdings unberücksichtigt (vgl. Neuberger 1997, S. 173). Entsprechend können die Qualitätsindikatoren die politischen und sozialen Funktionen der Medien nicht umfassend abdecken; ein direkter Rückschluss von der Bewertung der Medienqualität auf die Erfüllung der Funktionen ist nicht vollständig zulässig. Dies gilt ebenso für das Medienvertrauen, denn Vertrauen ist eine in die Zukunft gerichtete Erwartung (vgl. Kohring 2004, S. 120-121). Zudem umfasst die geläufige Operationalisierung von Medienvertrauen nach Kohring und Matthes (2004) nur einen Teil der Leistungen des Journalismus. Betrachtet man jedoch die im öffentlichen Diskurs geäußerten Kritikpunkte, so fällt auf, dass ganz diverse politische und soziale Funktionen der Medien berührt werden (vgl. z. B. Prochazka und Schweiger 2016). Der Lügenpresse-Vorwurf unterstellt, dass die Medien ihr Publikum nicht neutral und objektiv informieren, sondern bewusst Unwahrheiten verbreiten. Anhänger populistischer Bewegungen und Parteien fühlen ihre Interessen von den „Mainstream-Medien“ missachtet. Journalist*innen wird vorgeworfen, als Teil der Elite zu agieren, anstatt Politik und Wirtschaft zu kritisieren und kontrollieren.

Wie sehr diese Leistungen von den Medien erbracht werden, lässt sich durch die Einschätzung diverser Attributoren erheben, beispielsweise durch Journalist*innen, Wissenschaftler*innen, Medienaufsicht oder Rezipient*innen. Letztere stehen im Mittelpunkt dieses Beitrags. Er fragt danach, inwiefern die Bevölkerung solche Leistungen erfüllt sieht (,Performanzbewertung“) und welche individuellen Faktoren diese Wahrnehmung erklären können. Im Folgenden werden zunächst die normativ zugeschriebenen Funktionen der Medien theoretisch beschrieben. Im Anschluss werden der Forschungsstand aufgearbeitet und die Performanzbewertungen der Rezipient*innen anhand einer Repräsentativbefragung der deutschen Bevölkerung ab 18 Jahren untersucht und diskutiert.

\section{Soziale und politische Funktionen der Medien aus normativer Sicht}

Öffentlichkeit gehört zu den wichtigsten Voraussetzungen für das Bestehen von Demokratien (vgl. z. B. Martinsen 2009). Da Medien in modernen Demokratien die zentralen Instrumente sind, die Öffentlichkeit herstellen, ergeben sich an sie hohe normative Ansprüche. Aus normativer Perspektive werden die Funktionen der Medien folglich von ihrer demokratietheoretisch verankerten öffentlichen Aufgabe abgeleitet (vgl. Norris 2000; Strömbäck 2005). Je nach zugrunde liegendem Demokratiemodell erhalten die Funktionen der Medien dabei unterschiedliche Prioritäten (vgl. dazu z. B. Martinsen 2009). 


\subsection{Politische Funktionen}

Die Informationsfunktion gilt als Grundlage für alle weiteren Funktionen (vgl. Strömbäck 2005, S. 341), denn die Medien stellen in Demokratien eine wichtige Informationsplattform dar und konstruieren so die Wirklichkeitsvorstellungen der Bürger*innen. Aus der Bedeutung der Informationsfunktion resultieren auch die normativen Ansprüche an die Qualität der Berichterstattung wie Vollständigkeit oder Glaubwürdigkeit (vgl. Wellbrock und Klein 2014, S. 391).

Eine weitere zentrale Funktion der Medien besteht darin, Öffentlichkeit herzustellen, indem Informationen für die Gesellschaft jedermann zugänglich gemacht werden, um einen Informationsaustausch zwischen politischen und gesellschaftlichen Organisationen und der Bevölkerung zu ermöglichen (vgl. Graber 2003, S. 143; Ronneberger 1974, S. 199-201). In Zusammenhang mit der Herstellung von Öffentlichkeit steht die Artikulationsfunktion der Medien. Damit ist die Erwartung an die Medien gemeint, die Interessen der Bürger*innen zu artikulieren und somit als deren Sprachrohr zu fungieren (vgl. Schweiger 2017, S. 10). Medien gelten in modernen Demokratien daher als wichtigstes Forum für einen politischen Austausch zwischen Bürger*innen und politischen und gesellschaftlichen Organisationen und Institutionen (vgl. z. B. Schudson 2008, S. 12). Hohe Relevanz wird auch der politischen Meinungs- und Willensbildungsfunktion der Medien zugeschrieben. Medien sollen die Bürger*innen in die Lage versetzen, ein politisches Urteilsvermögen und eine eigene politische Haltung zu entwickeln und diese im politischen Prozess einzusetzen (vgl. Saxer 1974, S. 31).

Die Kritik- und Kontrollfunktion gilt ebenfalls als zentrale Leistung der Medien. Die Veröffentlichung von Kritik führt indirekt zu einer Kontrolle gesellschaftlicher Akteure. Daher wird auch von der Wächterfunktion (englisch: watchdog) gesprochen (vgl. Norris 2000, S. 28-29; Schudson 2008, S. 14-16; Schweiger 2017, S. 9-10). Ziel der politischen Sozialisations- und Bildungsfunktion ist es, durch die Vermittlung pluralistisch-demokratischer Werte das gesellschaftliche Zusammenleben zu ermöglichen, alle Bürger*innen zu integrieren und sie bei der Ausübung ihrer demokratischen Rechte und Pflichten zu unterstützen (vgl. Ronneberger 1974, S. 201-202; Voltmer 1999).

\subsection{Soziale Funktionen}

Im Wesentlichen lassen sich drei zentrale soziale Funktionen der Medien identifizieren. In Abgrenzung zur politischen Sozialisationsfunktion ist die allgemeine Sozialisationsfunktion weiter gefasst: Die Medien sollen demnach ein übergreifendes Normenbewusstsein der Rezipient*innen schaffen oder verstärken. Eng mit der Sozialisationsfunktion verknüpft ist die Orientierungsfunktion. Damit wird die Leistung der Medien angesprochen, Bürger*innen mit Informationen zu versorgen, die sie dabei unterstützen sollen, sich in ihrer Umwelt zurechtzufinden sowie Lösungsansätze für gesellschaftliche Probleme anzubieten (vgl. Burkart 2019, S. 329-330).

Nach der Funktion der sozialen Integration sollen Medien dafür sorgen, dass sich die Bürger*innen der Gesellschaft zugehörig fühlen und sich mit ihr identifizieren. Dies soll erreicht werden, indem die Medien gesellschaftlich anerkannte Verhaltens- 
weisen und -normen vermitteln und zur Akzeptanz dieser Normen beitragen (vgl. Burkart 2019, S. 330-333). ${ }^{1}$

\section{Soziale und politische Funktionen der Medien aus Sicht der Bürger*innen}

In der Literatur wird angenommen, dass das Publikum die Funktionen der Medien je nach seiner Rolle als Rezipient unterschiedlich wahrnimmt. Ist das Publikum Konsument, dann interessiert es sich für die Maximierung des individuellen Nutzens; die Kriterien entsprechen dann den Gratifikationserwartungen wie Unterhaltung oder Entspannung. Hier werden in der Regel Gratifikationen einzelner Medienangebote untersucht und nicht journalistischer Medien insgesamt. Dies geschieht dagegen, wenn Rezipient*innen als Bürger*innen eine gesamtgesellschaftliche Perspektive einnehmen (vgl. Hasebrink 2011; Herzog et al. 2006; Neuberger 2011). ${ }^{2}$ Die Erwartungen, die das Publikum dabei an die Medien hat, lassen sich von den Anforderungen ableiten, die sich aus den verschiedenen Modellen der Demokratietheorie für die politische Öffentlichkeit ergeben, wie z. B. die Erwartung einer vielfältigen Berichterstattung (vgl. Neuberger 2018, S. 18). Die normativen Funktionen journalistischer Medien können aus dieser Perspektive somit als Leistungen aufgefasst werden, die sie für die Gesellschaft erbringen. Das wird im Folgenden auch als Performanz der Medien bezeichnet. Somit lassen sich zunächst die Erwartungen der Bürger*innen an die Medienleistungen betrachten, aus denen sich ihre Performanzbewertungen speisen (vgl. Neuberger 2014, S. 233; Wolling 2004, S. 174).

\subsection{Erwartungen der Bürger*innen}

Das Publikum wurde in der Qualitätsforschung lange vernachlässigt. Im Vordergrund standen die Einschätzungen von Expert*innen, die Perspektive der Journalist*innen (vgl. z. B. Hanitzsch 2011; Weischenberg et al. 2006) oder Qualitätsanalysen der Berichterstattung durch Inhaltsanalyen (vgl. z. B. Arnold und Wagner 2018; Hagen 1995). ${ }^{3}$ Die konkreten Erwartungen der Rezipient*innen wurden in bisherigen Studien vornehmlich anhand der normativen Funktionen, anhand des journalistischen

\footnotetext{
1 Die meisten hier erwähnten Funktionen sind gesetzlich nicht vorgeschrieben (vgl. z. B. Pürer 2014, S. 422), aber eng mit den verfassungsmäßigen Aufgaben der Medien verknüpft, wie sie im Grundgesetz, in den Urteilen des Bundesverfassungsgerichts, in den Landespressegesetzen und im Grundversorgungsauftrag des öffentlich-rechtlichen Rundfunks festgeschrieben sind.

2 Dies steht im Einklang mit dem Public Value-Ansatz, der die Rezipient*innen nicht nur als Konsument*innen (consumer value), sondern auch als Bürger*innen erachtet und entsprechend den Beitrag für die gesamte Gesellschaft (citizen value) untersucht (vgl. BBC 2004).

3 Es wurde häufig in Frage gestellt, ob das Publikum aufgrund fehlender Kompetenz und mangelnden Einblicks überhaupt in der Lage ist, ein solches Qualitätsurteil zu fällen. Hintergrund ist das häufig beobachtete Spannungsverhältnis zwischen Qualität und Quote. Allerdings gibt es Hinweise, dass den Nutzer*innen durchaus bewusst ist, dass sie sich Angeboten zuwenden, die aus normativer Sicht nicht als qualitätvoll gelten (vgl. Herzog et al. 2006, S. 402), sowie Hinweise darauf, dass Qualitätsurteile durchaus einen Einfluss auf die Nutzung haben (vgl. Wolling 2002). Die Differenzierung zwischen Rezipient*innen als Konsument*innen und als Bürger*innen kann dieses Spannungsverhältnis auflösen, da aus der Bür-
} 
Rollenverständnisses oder der Qualitätskriterien operationalisiert. Peifer (2018) entwickelte eine Skala zur perceived news media importance, die sowohl die individuelle als auch die gesamtgesellschaftliche Bedeutung von sechs Funktionen umfasst (Information, Investigation, Analyse, Soziale Empathie, öffentliches Forum und Mobilisierung). Van der Wurff und Schönbach (2014) zeigen für die niederländische Bevölkerung, dass die diversen journalistischen Rollenmodelle und Werte wie beispielsweise die Aufgabe, die Regierung kritisch zu beobachten - von den Bürger*innen durchweg erwartet werden (vgl. auch Lieske 2008). Die wichtigsten Qualitätskriterien von Tageszeitungen sind aus Sicht des Publikums Aktualität, ausführliche Berichterstattung und Unabhängigkeit (vgl. Arnold 2009, S. 383). Im Online-Journalismus stellten sich Glaubwürdigkeit, Sachlichkeit und Unabhängigkeit als die zentralen Qualitätsmerkmale heraus (vgl. Neuberger 2014, S. 241; vgl. auch Heider et al. 2005, S. 958; Loosen et al. 2013; Tsfati et al. 2006, S. 162).

\subsection{Bewertungen der Bürger*innen}

Die Medienbewertungen der Bürger*innen wurden weitaus häufiger untersucht. Bisherige Studien widmen sich dabei vornehmlich den Bewertungen von Medienqualität im Hinblick auf unterschiedliche Gattungen wie Radio (vgl. Vowe und Wolling 2004), Tageszeitungen (vgl. Arnold 2009), einzelne Fernsehsendungen (vgl. Gehrau 2008) oder Online-Medien (vgl. Neuberger 2014). Hinsichtlich der Leistungen journalistischer Medien im Ganzen zeigte eine vom Bayerischen Rundfunk in Auftrag gegebene Studie, dass die Mehrheit mit den abgefragten Leistungen der Medien recht zufrieden ist. Der Großteil der Befragten (81\%) ist der Ansicht, dass Medien die Themen veröffentlichen, die für die Gesellschaft wichtig sind. $64 \%$ stimmen zu, dass Medien politische und gesellschaftliche Missstände aufdecken. Allerdings sind mehr Befragte der Ansicht, dass die Medien das Establishment eher stützen, statt es zu kontrollieren (vgl. BR.de 2016; vgl. auch Newman et al. 2019, S. 48). Qualitätsurteile spielen zudem eine zentrale Rolle in der Begleitforschung der öffentlichrechtlichen Rundfunkanstalten (vgl. z. B. Schröter 2016).

Donsbach et al. (2009) erfragen meines Wissens als einzige sowohl die Erwartungen als auch die Bewertungen des Publikums systematisch im Vergleich und können so einen Saldo zwischen beiden Urteilen bilden (vgl. auch aber Arnold 2009; Gehrau 2008; Neuberger 2014). Dabei zeigt sich, dass Journalist*innen intoleranter gegenüber anderen Meinungen sind und sozial Benachteiligte weniger unterstützen, als die Befragten es von ihnen erwarten. Im Gegensatz dazu setzen sie häufiger ihre eigenen Bedürfnisse durch und verfügen über mehr Macht und Einfluss, als die Befragten es sich wünschen (S. 71-73; vgl. auch Schielicke et al. 2014).

Aufbauend auf den bisherigen Überlegungen fragt die vorliegende Studie, wie gut es den Medien aus Sicht der Bevölkerung gelingt, ihre sozialen und politischen Leistungen zu erfüllen (FF1).

ger*innenperspektive nicht die individuellen Interessen im Vordergrund stehen, sondern das gesellschaftliche Gemeinwohl und gesamtgesellschaftliche Bedürfnisse (vgl. Peifer 2018; Serong 2015). 


\section{Einflüsse auf die wahrgenommene Performanz der Medien}

Studien, die sich mit Einflüssen auf die Bewertungen der Medienperformanz beschäftigen, sind rar. Hinweise liefert die Arbeit von Lieske (2008), in der höher Gebildete, politisch Interessierte und regelmäßige Zeitungsleser*innen die Funktionen der Medien am stärksten betonten, sich gleichzeitig aber auch am kritischsten über die Macht der Medien äußerten. Allerdings lassen sich aufgrund der oben beschriebenen Nähe zu den Konzepten Medienvertrauen und -qualität aus diesen Forschungsbereichen Annahmen ableiten. ${ }^{4}$ Die Einflussfaktoren lassen sich dabei in politische und medienbezogene Merkmale der Rezipient*innen unterteilen.

Im Hinblick auf politische Prädispositionen erwies sich Vertrauen in Politik als ein zentrales Korrelat zu Medienvertrauen (vgl. z. B. Hanitzsch et al. 2018; Ziegele et al. 2018). Zudem zeigen Studien, dass neben dem Vertrauen die Zufriedenheit mit den Leistungen des politischen und wirtschaftlichen Systems eine Rolle spielt (vgl. Bennett et al. 2001; van Eimeren et al. 2017). Für die Betrachtung der Medienleistungen wird daher an dieser Stelle zusätzlich das Vertrauen in die Wirtschaft herangezogen. Es lässt sich demzufolge annehmen: Je mehr Rezipient*innen Politik und Wirtschaft vertrauen (HIa), je zufriedener Rezipient*innen mit Politik und Wirtschaft sind (H1b), desto besser bewerten sie die Leistungen der Medien.

Die medienbezogenen Merkmale umfassen die Mediennutzung, d. h. das individuelle Medienrepertoire, sowie die Einstellungen gegenüber den Medien. Zahlreiche Studien weisen darauf hin, dass die Nutzung traditioneller Massenmedien, insbesondere des öffentlich-rechtlichen Rundfunks, mit einem höheren Medienvertrauen einhergeht (vgl. Jackob 2010; Kiousis 2001; Schultz et al. 2017; Tsfati und Cappella 2003; Ausnahme: Ladd 2012). Gil de Zúñiga und Hinsley (2013) zeigten zudem, dass mit steigender Nutzung traditioneller Medien ein positiveres Bild von ,gutem Journalismus" einhergeht. Medienvertrauen hängt darüber hinaus positiv mit einer einstellungskongruenten Mediennutzung zusammen (vgl. Arceneaux et al. 2012; Johnson und Kaye 1998).

Im Hinblick auf die Wahrnehmung der Berichterstattung gelten die beiden Wahrnehmungsphänomene Hostile media perception $(\mathrm{HMP})^{5}$ und Presumed media influence (PMI) ${ }^{6}$ als relevante Prädiktoren von medienbezogenen Einstellungen und Verhaltensweisen (vgl. z. B. Barnidge und Rojas 2014; Cohen et al. 2008). So zeigte die Forschung zur HMP, dass Personen mit ausgeprägten politischen Positionen die Medienberichterstattung stärker zuungunsten ihrer eigenen Position verzerrt wahrnehmen (vgl. Gunther und Liebhart 2005; Tsfati und Cohen 2005). Darauf aufbauend lässt sich vermuten, dass solche Wahrnehmungen sich negativ auf die Performanzbewertungen auswirken werden, da aus dieser Perspektive Funktionen wie eine neutrale Informationsquelle oder Sprachrohr aller Bürger*innen von den

\footnotetext{
${ }^{4}$ Im Hinblick auf die Medienqualität wurden jedoch vorwiegend Einflüsse auf die Erwartungen an die Qualität untersucht (z. B Arnold 2009), die sich nicht direkt auf die Bewertungen übertragen lassen. Daher wird im Folgenden stärker auf die Vertrauensliteratur zurückgegriffen.

5 Die HMP besagt, dass Individuen dazu tendieren, Medienberichte gegenüber der eigenen Meinung verzerrt wahrzunehmen (vgl. Vallone et al. 1985).

${ }^{6}$ PMI umfasst den Einfluss der Medien, den Individuen auf anderen Personen wahrnehmen.
} 
Medien vermutlich in geringerem Umfang geleistet werden. Ebenso ist ein Zusammenhang zwischen PMI und einer erfolgreichen Erfüllung der Medienfunktionen naheliegend, da ein gewisser Einfluss der Medien auf das Publikum als Voraussetzung für die Erfüllung der gesellschaftlichen Funktionen angenommen werden kann. Nur so können sie beispielsweise zur Meinungsbildung beizutragen oder das Interesse der Bürger*innen an Politik wecken. Demnach wird angenommen: Je intensiver Rezipient*innen etablierte Medien nutzen (H2a), je stärker Rezipient*innen Medien kongruent zur eigenen Meinung nutzen (H2b), je weniger Rezipient*innen die Berichterstattung gegenüber der eigenen Meinung verzerrt wahrnehmen $(\mathrm{H} 2 \mathrm{c})$, je mehr Einfluss Rezipient*innen den Medien beimessen (H2d), desto besser bewerten sie die Leistungen der Medien.

Im Kontext einer sich rasant verändernden Medienlandschaft gewinnt das Konzept der Informationsüberlastung zunehmend an Bedeutung. ${ }^{7}$ Es lässt sich vermuten, dass negative Gefühle der Überlastung, die mit der Medienberichterstattung verbunden werden, dazu führen, dass den Medien insgesamt weniger positive Leistungen zugeschrieben werden. Umgekehrt lässt sich mit der Media-dependency-Theorie (vgl. z. B. Ball-Rokeach und DeFleur 1976) argumentieren, dass eine hohe empfundene Abhängigkeit von den etablierten Medien in eine positivere Bewertung der Medienfunktion mündet (vgl. Jackob 2010). Zusammengefasst resultiert dies in folgende Hypothesen: Je niedriger die wahrgenommene Informationsüberlastung der Rezipient*innen ist (H2e) und je stärker sich Rezipient*innen von etablierten Medien abhängig fühlen (H2f), desto besser bewerten sie die Leistungen der Medien.

Nur wenn Medien autonom von Staat und Wirtschaft sind, können sie diese unabhängig kritisieren und damit kontrollieren. Um das Publikum objektiv zu informieren, sollte darüber hinaus gewährleistet sein, dass die Medieneigentümer keinen direkten Einfluss auf die Berichterstattung nehmen. Daraus leiten sich die letzten Hypothesen ab: Je autonomer Rezipient*innen die Medien von Politik (H3a), Wirtschaft (H3b) und Medieneigentümern (H3c) wahrnehmen, desto besser bewerten sie die Leistungen der Medien.

Recht wenig Aufmerksamkeit in der Vertrauensforschung hat bislang der Einfluss von Medienkompetenz erhalten, obwohl Dimensionen der Medienkompetenz wie Medienkunde und Medienreflexion als relevante Prädiktoren für bürgerschaftliche Kompetenzen erachtet werden und die Bürger*innen befähigen sollten, die Medienperformanz besser einzuschätzen (vgl. z. B. Martens und Hobbs 2015). So konnte Voigt (2016) zeigen, dass Medienkompetenz bei einigen Qualitätsdimensionen einen Einfluss auf die Fähigkeit zur Qualitätswahrnehmung hat. Lieske zeigte indes (2008), dass kompetentere Befragte sich besonders kritisch über die Medien äußerten. Es stellt sich daher folgende Forschungsfrage: Wie sehr beeinflusst der Grad der Medienkompetenz die Zufriedenheit mit den Medienleistungen (FF2)?

\footnotetext{
7 Da Individuen vermutlich nur über begrenzte Kapazitäten zur Informationsaufnahme verfügen, beschreibt es den Zustand, in dem Personen die ihnen zur Verfügung stehenden Informationen nicht vollständig aufnehmen können (z. B. Rogers und Agarwala-Rogers 1975).
} 


\section{Methode}

Antworten auf die Forschungsfragen und Hypothesen sollte im Dezember 2016 und Januar 2017 eine computergestützte, repräsentative Telefonbefragung (CATI) der deutschen Bevölkerung ab 18 Jahren $(N=1005)$ geben (Dual-Frame-Ansatz; Stichprobe: $70 \%$ Festnetz, $30 \%$ Mobilfunk, ungewichtet). Die Items zu den Erwartungen des Publikums wurden theoretisch hergleitet und in einer nicht-repräsentativen Vorstudie getestet (Online-Befragung, $N=559$ ). Hier zeigte sich, dass alle Befragten die vorgelegten Medienleistungen im Durchschnitt auch weitgehend erwarten (Mittelwerte zwischen 3,2 [Integrationsfunktion] und 4,8 [Informationsfunktion], 5-stufige Skala). Es ist nun das Ziel dieser Studie zu überprüfen, inwiefern es den Medien aus Sicht der Rezipient*innen gelingt, diese Leistungen tatsächlich zu erfüllen. Dabei wird zunächst keine Differenzierung zwischen den Mediengattungen vorgenommen, sondern es werden analog zur normativen Betrachtungsweise die Funktionen der Massenmedien im Allgemeinen untersucht.

\subsection{Stichprobe}

Die Hälfte der Befragten ist männlich (50\%). Im Durchschnitt sind die Teilnehmer 53 Jahre alt. $38 \%$ verfügen über einen (Fach-)Hochschulabschluss, bei $20 \%$ ist das (Fach-)Abitur der höchste Bildungsabschluss. $17 \%$ verfügen über eine abgeschlossene Lehre, $16 \%$ über einen Realschul-, $9 \%$ über einen Hauptschulabschluss. 14,5\% der Befragten haben einen Migrationshintergrund.

\subsection{Abhängige Variablen}

Aus den theoretischen Vorüberlegungen und der Vorstudie wurden elf Items zur Bewertung der Medienleistungen entwickelt, die die zentralen politischen und sozialen Funktionen der Medien abbilden (vgl. Fawzi und Fortkord 2017). Die Performanzbewertungen orientieren sich an den Items zum journalistischen Rollenselbstverständnis (vgl. Weischenberg et al. 2006) sowie an den normativen Erwartungen an die Medien (vgl. Abschn. 2). Ausgangspunkt waren entsprechend die Informationsfunktion, Herstellung von Öffentlichkeit, Artikulations-Meinungs- und Willensbildungs-, Kritik- und Kontroll-, politische Sozialisations- und Bildungs-, Sozialisations- und Orientierungsfunktion sowie die Funktion der sozialen Integration. Jede Bewertung wurde dabei mit einem bzw. zwei Items erfasst, wenn die jeweilige Leistung zwei Bereiche beinhaltet (z.B. Kritik und Kontrolle). Die Befragten gaben an, inwiefern es ihrer Ansicht nach den Medien gelingt, diese jeweilige Leistung zu erfüllen (5stufige Skala, 1= gelingt den Medien überhaupt nicht, 5= gelingt den Medien voll und ganz), beispielsweise „Politik und Wirtschaft zu kritisieren“ oder „Sprachrohr der Bürger zu sein“ (vgl. Tab. 1). Eine explorative Faktorenanalyse ermittelte eine Ein-Faktor-Lösung (Maximum-Likelihood, Varimax-Rotation), so dass die elf Items für die Analyse von Einflussfaktoren zu einem Mittelwertindex zusammengefasst wurden, der die abhängige Variable abbildet $(M=3,1 ; S D=0,66$; Cronbachs $\alpha=0,88)$. 
Tab. 1 Publikumsbewertungen der sozialen und politischen Funktionen der Medien in Deutschland

\begin{tabular}{|c|c|c|c|c|c|}
\hline $\begin{array}{l}\text { „Wie gut gelingt es den tradi- } \\
\text { tionellen Medien in Deutsch- } \\
\text { land Ihrem Eindruck nach, die } \\
\text { folgenden Funktionen zu erfül- } \\
\text { len?“ }\end{array}$ & $\begin{array}{l}\text { Gelingt den } \\
\text { Medien } \\
\text { (überhaupt) } \\
\text { nicht (in \%) }\end{array}$ & $\begin{array}{l}\text { Teils/ } \\
\text { teils } \\
\text { (in \%) }\end{array}$ & $\begin{array}{l}\text { Gelingt den } \\
\text { Medien } \\
\text { (voll und } \\
\text { ganz) (in \%) }\end{array}$ & $\begin{array}{l}\text { Kann ich } \\
\text { nicht beur- } \\
\text { teilen/k. A. } \\
\text { (in \%) }\end{array}$ & $\begin{array}{l}M \\
(\mathrm{SD})\end{array}$ \\
\hline $\begin{array}{l}\text { „Politische Missstände und } \\
\text { Skandale aufzudecken“ } \\
\text { (Kontrolle) }\end{array}$ & 17 & 27 & 54 & 2 & $\begin{array}{l}3,5 \\
(1,1)\end{array}$ \\
\hline $\begin{array}{l}\text { „Zur Meinungsbildung der } \\
\text { Bürger beizutragen“ } \\
\text { (Meinungs- und Willensbil- } \\
\text { dung) }\end{array}$ & 14 & 33 & 51 & 2 & $\begin{array}{l}3,5 \\
(1,0)\end{array}$ \\
\hline $\begin{array}{l}\text { „Politik und Wirtschaft zu kriti- } \\
\text { sieren“ } \\
\text { (Kritik) }\end{array}$ & 18 & 36 & 45 & 2 & $\begin{array}{l}3,4 \\
(1,0)\end{array}$ \\
\hline $\begin{array}{l}\text { „Komplexe Sachverhalte zu } \\
\text { erklären und zu vermitteln“ } \\
\text { (Information/Interpretation) }\end{array}$ & 20 & 39 & 39 & 2 & $\begin{array}{l}3,2 \\
(1,0)\end{array}$ \\
\hline $\begin{array}{l}\text { „Die Bürger über Ereignisse aus } \\
\text { Politik und Gesellschaft neutral } \\
\text { und präzise zu informieren“ } \\
\text { (Information) }\end{array}$ & 24 & 40 & 34 & 2 & $\begin{array}{l}3,1 \\
(1,0)\end{array}$ \\
\hline $\begin{array}{l}\text { „Das Interesse an politischen } \\
\text { Fragen zu wecken“ } \\
\text { (politische Sozialisation) }\end{array}$ & 27 & 38 & 32 & 2 & $\begin{array}{l}3,1 \\
(1,0)\end{array}$ \\
\hline $\begin{array}{l}\text { „Die Interessen von Benach- } \\
\text { teiligten in der Bevölkerung zu } \\
\text { berücksichtigen“" } \\
\text { (Integration) }\end{array}$ & 30 & 39 & 28 & 3 & $\begin{array}{l}3,0 \\
(1,0)\end{array}$ \\
\hline $\begin{array}{l}\text { „Zwischen Politik und Gesell- } \\
\text { schaft zu vermitteln“ } \\
\text { (Herstellung von Öffentlichkeit) }\end{array}$ & 25 & 49 & 22 & 3 & $\begin{array}{l}3,0 \\
(0,9)\end{array}$ \\
\hline $\begin{array}{l}\text { „Ein demokratisches Verständ- } \\
\text { nis zu schaffen, das die gesamte } \\
\text { Gesellschaft verbindet“ } \\
\text { (Sozialisation, Integration) }\end{array}$ & 31 & 41 & 24 & 3 & $\begin{array}{l}2,9 \\
(1,0)\end{array}$ \\
\hline $\begin{array}{l}\text { „Sprachrohr der Bürger zu sein“ } \\
\text { (Artikulation) }\end{array}$ & 34 & 43 & 21 & 2 & $\begin{array}{l}2,8 \\
(1,0)\end{array}$ \\
\hline $\begin{array}{l}\text { „Lösungsvorschläge für die } \\
\text { Probleme der Gesellschaft } \\
\text { anzubieten“ } \\
\text { (Orientierung) }\end{array}$ & 41 & 39 & 17 & 2 & $\begin{array}{l}2,7 \\
(1,0)\end{array}$ \\
\hline $\begin{array}{l}\text { „Alles in allem erfüllen die } \\
\text { traditionellen Medien ihre Auf- } \\
\text { gabe in der demokratischen } \\
\text { Gesellschaft.“a }\end{array}$ & 9 & 29 & 61 & 2 & $\begin{array}{l}3,7 \\
(1,0)\end{array}$ \\
\hline
\end{tabular}

$N=988-1004$

„Ist keine Aufgabe der Medien“ bleibt in der Tabelle unberücksichtigt (jeweils zwischen 0 und $2 \%$ ) 5-stufige Skala, die beiden äußeren Skalenpunkte wurden zusammengefasst

aAtwortskala: $1=$ trifft überhaupt nicht zu bis $5=$ trifft voll und ganz zu 


\subsection{Unabhängige Variablen}

Die politischen Merkmale umfassten das politische Interesse der Befragten (5-stufige Skala, $1=$ überhaupt nicht, $5=$ sehr stark; $M=4,02 ; S D=0,97)$, ihre Zufriedenheit mit der Demokratie in Deutschland $(M=3,27 ; S D=1,03)$ und der derzeitigen Wirtschaftslage $(1=$ überhaupt nicht zufrieden, $5=$ voll und ganz zufrieden; $M=3,62 ; S D=0,95$ ), Politikvertrauen (Mittelwertindex aus Vertrauen in Bundesregierung, Parteien und Politiker, 1=kein Vertrauen, 5= sehr großes Vertrauen; $M=2,64 ; S D=0,89 ; \alpha=0,85)$ und Vertrauen in Wirtschaftsunternehmen (gleiche Skala; $M=2,45 ; S D=0,96)$.

Die Mediennutzung umfasste die Nutzung acht verschiedener Mediengattungen zur politischen Information (öffentlich-rechtlicher Rundfunk, privater Rundfunk, Lokalzeitungen, deren Online-Angebote, soziale Netzwerke etc. $(0=$ nie, $1=$ ein Tag pro Woche bis $7=$ sieben Tage pro Woche) $).{ }^{8}$ Zudem wurde die selektive Mediennutzung abgefragt (,Ich nutze hauptsächlich Medien und Nachrichten, die mit meiner eigenen Einstellung übereinstimmen“, $M=2,65 ; S D=1,3$ ).

Die Hostile-Media-Wahrnehmung wurde aus der Differenz zwischen der eigenen politischen Position und der wahrgenommenen politischen Position der etablierten Medien (jeweils $0=$ links bis $10=$ rechts) gebildet. Negative Werte wurden umgekehrt, so dass die Skala Werte von 0-10 umfasst $(M=1,46 ; S D=1,68)$. Presumed media influence erhielt ein Item („Die Berichterstattung hat einen großen Einfluss auf die Meinung der Bevölkerung“; vgl. z. B. Cohen et al. 2008; $M=4,13$; $S D=0,86$ ), ebenso wie Informationsüberlastung (,Die Menge an Informationen aus den Medien überlastet mich“; vgl. z. B. Beaudoin 2008; $M=2,38 ; S D=1,29$ ). Die Mediendependenz wurde anhand einer Frage zur Rolle der Medien bei der Meinungsbildung abgefragt (,Um mir eine Meinung zu bilden, bin ich bereit, mich auf die Berichterstattung der traditionellen Medien zu verlassen“). Schließlich enthielt der Fragebogen drei Items zur Selbsteinschätzung der Dimension Medienkunde, die selbst entwickelt wurden (z.B. „Ich bin gut über die Aufgaben informiert, die der Journalismus in Demokratien erfüllen soll“, „Ich bin gut über aktuelle Entwicklungen in der deutschen Medienlandschaft informiert"; $M=3,54 ; S D=0,81 ; \alpha=0,62$ ). Die Zustimmung zu diesen Items wurde auf 5-stufigen Skalen erhoben $(1=$ trifft überhaupt nicht zu, 5= trifft voll und ganz zu). Zur Messung der Autonomie der Medien dienten drei Items zum Einfluss von Politik, Wirtschaft und Medieninhabern auf die Medienberichterstattung ( $1=$ überhaupt keinen Einfluss, $5=$ sehr großen Einfluss).

Als Kontrollvariablen wurden das Alter in Jahren, das Geschlecht, der Bildungsgrad, der Migrationshintergrund (falls die Person oder mindestens ein Elternteil im Ausland geboren wurde) und das Bundesland (recodiert nach West- und Ostdeutschland) erhoben.

\footnotetext{
8 Die Nutzung von Alternativmedien wurde ebenfalls abgefragt, allerdings gaben $15 \%$ an, diese nicht zu kennen, weitere $80 \%$ nutzen sie nie. Aus diesem Grund wurden sie aus der Analyse ausgeschlossen.
} 


\section{Ergebnisse}

Wie bewerten Rezipient*innen die den Medien normativ zugeschriebenen Leistungen $(F F 1)$ ? An erster Stelle positionieren sie die Kritik- und Kontrollfunktion. Etwas mehr als die Hälfte der Befragten (54\%) ${ }^{9}$ ist der Ansicht, dass die Medien politische Missstände und Skandale aufdecken. 16\% finden dagegen, dass dies den Medien nicht gelingt. Weiterhin leisten die Medien $45 \%$ der Befragten zufolge eine kritische Beobachtung von Politik und Wirtschaft. 18\% nehmen dies nicht wahr. Ein ähnliches Bild zeigt sich hinsichtlich der Meinungsbildungsfunktion. Die Hälfte der Befragten (51\%) findet, dass die Medien zur Meinungsbildung der Bürger*innen beitragen. $14 \%$ zufolge gelingt dies den Medien nicht.

Ihre Informationsfunktion, eine der grundlegenden Leistungen, erfüllen die Medien nach Ansicht von mehr als einem Drittel der Befragten ausreichend. So finden $39 \%$, dass es den Medien gelingt, komplexe Sachverhalte zu erklären und zu vermitteln. Jeder Fünfte (20\%) findet nicht, dass die Medien in Deutschland diese Leistung angemessen ausüben. Die Nutzer*innen über aktuelle Ereignisse möglichst neutral und präzise zu informieren gelingt den Medien nach Ansicht der Befragten ebenfalls nur mäßig. Jeder Dritte (34\%) äußert sich hierbei zufrieden, jeder Vierte (24\%) ist mit der Umsetzung dieser Leistung nicht zufrieden. Eine recht ähnliche Verteilung findet sich hinsichtlich der politischen Sozialisationsfunktion: Gemäß einem Drittel der Befragten (32\%) wecken Medien das Interesse der Bürger*innen für politische Fragen; knapp ein weiteres Drittel hält diese Funktion für nicht erfüllt (27\%).

Um die soziale Integration aller Bürger*innen sicherzustellen, sollen die Medien insbesondere Minderheiten und benachteiligte Gruppen berücksichtigen. 28\% der Befragten sind der Ansicht, dass die Medien dies in ihrer Berichterstattung umsetzen, knapp jeder Dritte (30\%) äußert sich diesbezüglich unzufrieden mit den Medien. Die Funktion der Herstellung von Öffentlichkeit beinhaltet den Anspruch an die Medien, den Austausch zwischen Bürger*innen und Politik sicherzustellen. Dies gelingt den Medien jedem fünften Befragten zufolge (22\%), jeder Vierte $(25 \%)$ hat einen negativen Blick auf diese Medienleistung. Dass die Medien durch ihre Berichterstattung ein demokratisches Grundverständnis schaffen, das die gesamte Gesellschaft verbindet (Sozialisations- und Integrationsfunktion), äußert jeder vierte Befragte (24\%). Knapp jeder Dritte (31\%) ist nicht der Ansicht, dass die Medien auf diesem Weg zur sozialen Integration beitragen.

Eine Erfüllung der Artikulationsfunktion, d.h. Sprachrohr der Bürger*innen zu sein, attestiert jeder Fünfte (21\%) Befragte den Medien. Jeder Dritte (34\%) findet nicht, dass die Medien mit ihrer Berichterstattung diesem Anspruch genügen. Am wenigsten Befragte beobachten, dass die Medien in der Lage sind, Lösungsvorschläge für die Probleme der Gesellschaft anzubieten (Orientierungsfunktion). 17\% sind hier mit den Medien zufrieden, $41 \%$ zufolge erfüllen die Medien diese Funktion jedoch nicht.

Einen wesentlich positiveren Eindruck vermittelt die zusätzlich zu den konkreten Funktionen der Medien gestellte Frage, ob die Medien ihre demokratischen Auf-

\footnotetext{
${ }_{9}$ Die folgenden Prozentangaben umfassen, wenn nicht anders angegeben, die jeweils äußeren Skalenpunkte der 5-stufigen Skala.
} 
gaben im Allgemeinen erfüllen. Bemerkenswerterweise erhielt diese im Vergleich zu den konkreten Einzelleistungen mit Abstand die größte Zustimmung. $61 \%$ der Befragten sind demzufolge ,alles in allem“ zufrieden mit der Leistung der Medien, $29 \%$ zumindest teilweise, nur neun Prozent äußern sich unzufrieden.

Welche Faktoren hängen mit den Bewertungen der Medienperformanz zusammen? Dazu wurde mittels multipler linearer OLS-Regression der Einfluss diverser Rezipientenmerkmale auf die Bewertungen untersucht (blockweise, Tab. 2). Im ersten Block wurden die Kontrollvariablen eingeführt, von denen das Alter und der Wohnort einen signifikanten Einfluss haben. Der zweite Block beinhaltet die politischen Einstellungen der Befragten. Diese leisten einen deutlichen Beitrag zur Erklärung der Gesamtvarianz $\left(R^{2}=0,21\right)$. Politisches Interesse, Demokratiezufriedenheit, Politikvertrauen und Zufriedenheit mit der aktuellen Wirtschaftslage stehen in positivem Zusammenhang mit der Zufriedenheit mit den Medienleistungen. Hypothese 1a kann somit für das Politikvertrauen beibehalten werden, nicht jedoch für das Vertrauen in die Wirtschaft, das keinen Einfluss hat. Hypothese 1b kann sowohl für die Zufriedenheit mit der Demokratie als auch mit der aktuellen Wirtschaftslage bestätigt werden.

Die Mediennutzung differenziert nach den einzelnen Gattungen (Block 3) spielt bemerkenswerterweise keinerlei Rolle, womit Hypothese 2a abgelehnt werden muss. Die Nutzung von Boulevardmedien hat zunächst einen positiven Einfluss, doch dieser verschwindet im nächsten Modell. Eine Rolle spielt dagegen die Art der Mediennutzung. Je eher Rezipient*innen Medien nutzen, die mit ihren eigenen Einstellungen übereinstimmen, desto zufriedener sind sie mit den Leistungen der Medien $(H 2 b)$. Hinsichtlich der Einstellungen gegenüber den Medien zeigt sich folgender Zusammenhang: Je weniger stark die Befragten die Medien verzerrt im Vergleich zu ihrer eigenen politischen Einstellung wahrnehmen (HMP, H2c), desto besser bewerten sie die Medienperformanz. Ein positiver Zusammenhang besteht auch zur wahrgenommenen Mediendependenz $(H 2 f)$. Die empfundene Informationsüberlastung spielt dagegen keine Rolle, Hypothese $2 \mathrm{e}$ wird somit verworfen.

Im letzten Modell wurde schließlich die wahrgenommene Autonomie der Medien von Politik, Wirtschaft und Medieninhabern berücksichtigt. Alle drei Faktoren haben einen leicht negativen Einfluss, aber nur die Unabhängigkeit von Wirtschaftsunternehmen ist ein signifikanter Prädiktor: Mit steigender wahrgenommener Autonomie der Medien von der Wirtschaft wächst die Zufriedenheit der Befragten mit den Medienleistungen, was Hypothese $3 \mathrm{~b}$ bestätigt. Die Hypothesen 3a und 3c können hingegen nicht bestätigt werden. Zusätzlich sind im letzten Modell auch die PMI (Bestätigung von $H 2 d$ ) und die Medienkompetenz ( FF2) relevante Prädiktoren. D.h. je mehr Einfluss die Befragten den Medien auf die Bevölkerung zuschreiben und je medienkompetenter sie sich einschätzen, umso zufriedener sind sie. Von den Kontrollvariablen haben unter Berücksichtigung aller Faktoren die Bildung und der Wohnort einen Einfluss. Personen mit niedrigerer Bildung und Personen aus Westdeutschland bewerten die Leistungen der Medien besser. 
Tab. 2 Prädiktoren der Performanzbewertung

\begin{tabular}{|c|c|c|c|c|c|}
\hline & Modell 1 & Modell 2 & Modell 3 & Modell 4 & Modell 5 \\
\hline Konstante & $1,857 * * *$ & $0,698 * * *$ & $0,468^{*}$ & 0,285 & $0,596^{* *}$ \\
\hline Alter & $0,140 * * *$ & $0,072 *$ & 0,071 & 0,060 & 0,069 \\
\hline Geschlecht $($ männlich = 1) & $-0,016$ & 0,017 & 0,008 & $-0,010$ & $-0,003$ \\
\hline Bildung & 0,001 & $-0,091 * *$ & $-0,072 *$ & $-0,058$ & $-0,066^{*}$ \\
\hline Migrationshintergrund $(=1)$ & 0,039 & 0,023 & 0,024 & 0,037 & 0,042 \\
\hline Ostdeutschland $(=1)$ & $-0,089 *$ & $-0,062 *$ & $-0,063 *$ & $-0,065^{*}$ & $-0,066^{*}$ \\
\hline Korr. $\mathrm{R}^{2}$ & $0,022 * * *$ & & & & \\
\hline Politisches Interesse & & $0,090 * *$ & $0,099 * *$ & $0,104 * *$ & $0,102 * *$ \\
\hline Demokratiezufriedenheit & & $0,215 * * *$ & $0,220 * * *$ & $0,174 * * *$ & $0,165 * * *$ \\
\hline Politikvertrauen & & $0,236 * * *$ & $0,229 * * *$ & $0,145 * * *$ & $0,135 * * *$ \\
\hline Zufriedenheit Wirtschaftslage & & $0,079 *$ & $0,084^{*}$ & $0,075^{*}$ & $0,075^{*}$ \\
\hline Vertrauen in Wirtschaft & & 0,044 & 0,031 & 0,018 & 0,010 \\
\hline Korr. $\mathrm{R}^{2}$ & & $0,232 * * *$ & & & \\
\hline Öff.-rechtl. Rundfunk & & & 0,028 & $-0,017$ & $-0,013$ \\
\hline Privater Rundfunk & & & 0,005 & 0,006 & 0,011 \\
\hline Boulevardzeitungen & & & $0,067 *$ & 0,051 & 0,051 \\
\hline Lokalzeitungen & & & $-0,018$ & $-0,038$ & $-0,034$ \\
\hline $\begin{array}{l}\text { Überregionale Zeitungen/ } \\
\text { Magazine }\end{array}$ & & & $-0,015$ & $-0,028$ & $-0,034$ \\
\hline $\begin{array}{l}\text { Onlineangebote von Boulevard- } \\
\text { medien und priv. RF }\end{array}$ & & & 0,027 & 0,035 & 0,043 \\
\hline $\begin{array}{l}\text { Onlineangebote von Zeitungen, } \\
\text { Magazinen und ö-r RF }\end{array}$ & & & $-0,038$ & $-0,032$ & $-0,043$ \\
\hline $\begin{array}{l}\text { Von Freunden auf Soz. Netzwer- } \\
\text { ken geteilte Beiträge }\end{array}$ & & & $-0,016$ & $-0,026$ & $-0,025$ \\
\hline Selektive Mediennutzung & & & $0,102 * *$ & $0,089 * *$ & $0,087 * *$ \\
\hline Korr. $\mathrm{R}^{2}$ & & & $0,232 * * *$ & & \\
\hline Informationsüberlastung & & & & $-0,029$ & $-0,019$ \\
\hline Hostile Media Wahrnehmung & & & & $-0,167 * * *$ & $-0,149 * * *$ \\
\hline Presumed Media Influence & & & & 0,054 & $0,078 *$ \\
\hline Mediendependenz & & & & $0,242 * * *$ & $0,237 * * *$ \\
\hline Medienkompetenz & & & & 0,059 & $0,065^{\dagger}$ \\
\hline Korr. $\mathrm{R}^{2}$ & & & & $0,326 * * *$ & \\
\hline \multicolumn{6}{|l|}{ Autonomie der Medien von ... } \\
\hline ... Bundesregierung & & & & & $-0,042$ \\
\hline ... Wirtschaftsunternehmen & & & & & $-0,074 *$ \\
\hline ... Medieninhabern & & & & & $-0,034$ \\
\hline Korr. $\mathrm{R}^{2}$ & & & & & $0,336 * * *$ \\
\hline
\end{tabular}

$N=787$

OLS Regression mit listenweisem Fallausschluss standardisierte b-Werte

VIF zwischen 1,1 und 2,1

${ }^{* * *} p<0,001 ;{ }^{* *} p<0,01 ;{ }^{*} p<0,05 ; \dagger p<0,10$ 


\section{Diskussion}

Das Ziel dieses Beitrags war es, die umfangreiche Forschung zur Publikumsqualität zu erweitern und dabei mit der Analyse der politischen und sozialen Funktionen der Medien eine Abstraktionsebene höher anzusetzen. Denn die Qualitätskriterien leiten sich zwar von den normativen Medienfunktionen ab, sie lassen jedoch nur bedingt Rückschlüsse auf die Erfüllung dieser Funktionen zu. Um zu untersuchen, wie gut es den Medien gelingt, diese Leistungen für die Gesellschaft zu erbringen, wurden die konkreten Bewertungen der Medienperformanz durch die Bevölkerung von den normativen Funktionen der Medien wie Information oder Kritik und Kontrolle abgeleitet. Ein Großteil der Deutschen ist dabei recht zufrieden mit den Leistungen der Medien, es liegt insbesondere eine ,diffuse“ Zufriedenheit vor. Erst im Hinblick auf die konkreten Leistungen der Medien treten deutliche Differenzen auf. Während die Medien ihre Kritik- und Kontrollfunktion sowie die Informations- und Meinungsbildungsfunktion den Befragten zufolge relativ gut erfüllen, steigt der Anteil der Unzufriedenen hinsichtlich der Erfüllung der Integrations-, Artikulations- und Orientierungsfunktion deutlich an. Hier stellen die Befragten eindeutige Defizite fest.

Bei der Interpretation der Ergebnisse gilt es zu bedenken, dass es sich um die von den Rezipient*innen wahrgenommenen Leistungen der Medien handelt. Dadurch kann die teilweise große Diskrepanz innerhalb der Bewertungen erklärt werden. Politische und medienbezogene Voreinstellungen - und weniger das Mediennutzungsverhalten - sind die wichtigsten Prädiktoren für diese Bewertungen. Sie sind somit unabhängig davon, ob die Rezipient*innen eher dazu tendieren, öffentlich-rechtliches Fernsehen oder Boulevardmedien zu konsumieren; hinsichtlich der Mediennutzung entscheidet allein die Kontaktintensität mit einstellungskongruenten Medieninhalten. Übergreifend hat die Bereitschaft, sich bei der Meinungsbildung auf die Medien zu verlassen, den größten Einfluss; es folgen politische Einstellungen wie Demokratiezufriedenheit, Politikvertrauen, die Hostile-Media-Wahrnehmung und das politische Interesse. Im Vergleich zeigt sich, dass die Performanzbewertungen stärker durch politische als durch medienbezogene Merkmale der Befragten erklärt werden. Diese Ergebnisse bestätigen Studien, die zeigen, dass Einstellungsweisen gegenüber sowie Vertrauen in Medien und Politik zusammenhängen und nicht unabhängig voneinander betrachtet werden können. Bemerkenswerterweise hatte die wahrgenommene Autonomie der Medien von der Politik keinen Einfluss, nur von der Wirtschaft. ${ }^{10}$ Möglicherweise sind diese Einschätzungen bereits durch Demokratiezufriedenheit und Politikvertrauen mitberücksichtigt. Auch die empfundene Informationsüberlastung ist kein Prädiktor für die Leistungsbewertung, was daran liegen kann, dass dieses negative Gefühl nur von einem geringen Teil der Befragten empfunden wird.

Wo die Ergebnisse miteinander vergleichbar sind, stehen sie mit bisheriger Forschung weitgehend im Einklang. Beispielsweise werden die Kontrollfunktion in der Studie des Bayerischen Rundfunks ähnlich gut bewertet (vgl. BR.de 2016, S. 11)

\footnotetext{
10 Dies ist nicht als Tatsache zu interpretieren, dass die Medien von den beiden Systemen autonom wahrgenommen werden. Tatsächlich ist es so, dass die Mehrheit der Befragten eine sehr große Abhängigkeit der Medien von Politik (57\%) und Wirtschaft (68\%) beobachtet.
} 
und ebenso große Defizite in der Orientierungsfunktion festgestellt: Eine deutliche Mehrheit findet, dass die Medien zu viel über Probleme und zu wenig über Lösungen berichten. Tendenziell bestätigen die Befunde, dass ein Teil des Publikums im Hinblick auf die Leistungen der Medien enttäuscht ist, was ihnen vom Journalismus geboten wird (vgl. auch Donsbach et al. 2009).

In Zukunft gilt es insbesondere die unzufriedenen Bevölkerungsgruppen in den Blick zu nehmen. Denn hat ein Nutzer das Gefühl, dass die Medien nicht objektiv informieren, findet er seine Ansichten in keinen etablierten Medien wieder und sich damit gegenüber der Politik nicht repräsentiert oder ist er der Überzeugung, dass die Medien nicht in der Lage sind, Politiker und deren tagtäglichen Entscheidungen zu kritisieren, so kann dies gerade in Zeiten stärker werdender populistischer Kräfte die politische Teilhabe und den gesellschaftlichen Zusammenhalt negativ beeinflussen. Dies könnte eine Ursache für die zunehmende Abwendung dieser Bevölkerungsgruppe von den etablierten Medien hin zu Alternativmedien sein, die eine höhere Übereinstimmung zu den eigenen politischen Interessen aufweisen (die normativen Funktionen jedoch eher noch weniger erfüllen). Das wiederum birgt die Gefahr, dass diese Gruppen den gemeinsamen öffentlichen Diskurs verlassen, was eine Polarisierung der Gesellschaft verstärken könnte. Auch der positive Einfluss von Demokratiezufriedenheit und Politikvertrauen auf die Bewertungen der Medienleistungen verweist darauf, dass diejenigen, die sich bereits von der Politik vernachlässigt fühlen, diesen Eindruck auf die Medien übertragen, was eine weitere empfundene Ausgrenzung zur Folge haben kann. Ihre Vorstellungen von der demokratischen und gesellschaftlichen Rolle der Medien unterscheidet sich von anderen gesellschaftlichen Gruppen deutlich (vgl. Jandura und Kösters 2018; van Eimeren et al. 2017).

Die Studie unterliegt einigen Einschränkungen. So lassen die Befunde erstens keinen direkten Rückschluss darauf zu, welche konkreten normativen Erwartungen das Publikum an die Medien hat. Es lässt sich zwar argumentieren, dass die Bewertung der tatsächlichen Leistungen der Medien immer im Abgleich mit den individuellen Erwartungen erfolgt, doch es wäre sicherlich interessant gewesen, zusätzlich Kenntnisse über die Funktionen sowie die konkreten Erwartungen der Rezipient*innen zu erfragen. Ebenso wäre es interessant gewesen, zwischen unterschiedlichen Mediengattungen zu differenzieren. Hinsichtlich der Erklärungsfaktoren ist die niedrige Reliabilität der verwendeten Skala für Medienkompetenz festzuhalten sowie die Tatsache, dass Items wie Mediendependenz und Demokratievertrauen mit nur einem Item abgefragt wurden.

In Anlehnung an die Diskussion in der Qualitätsforschung muss an dieser Stelle diskutiert werden, wie mit den vorliegenden Publikumsbewertungen umzugehen ist. Auf der einen Seite plädieren Ansätze für eine starke Orientierung an den Publikumsinteressen, andere betrachten die Leistungen der Medien weitgehend unabhängig vom Publikum (vgl. zu verschiedenen Modellen Scholl et al. 2014) bzw. plädieren dafür, dass der Journalismus die Rezipienteninteressen durchaus verfehlen darf (vgl. Görke 2014). Darüber hinaus gibt es sicherlich einige Gründe dafür, den Ansatz dieser Studie und folglich die Ergebnisse im Hinblick auf ihre Validität kritisch zu betrachten. Es ist fraglich, ob den Befragten die gesellschaftlichen Medienfunktionen umfassend bekannt sind. Mit der Bewertung der Leistungen aller Medien für 
die gesamte Gesellschaft wurde den Befragten sehr viel abverlangt. Es kann nicht sichergestellt werden, dass die Befragten das gleiche Verständnis von Begriffen wie Gesellschaft oder Politik haben. Schließlich weisen die Befunde darauf hin, dass obwohl das Bezugsobjekt stets die gesamtgesellschaftlichen Leistungen waren - die Befragten diese nicht ausschließlich im Blick hatten, sondern tatsächlich die eigenen Interessen und Bedürfnisse bzw. die ihrer sozialen Gruppe in das Urteil eingegangen sind.

Die Ergebnisse dieser Befragung zeigen, dass die Rezipient*innen sich zumindest ein Urteil zutrauen; möglicherweise, indem sie von den von ihnen genutzten Angeboten sowie Gesprächen über Medien auf das Gesamtangebot abstrahieren. Ein sehr geringer Teil der Befragten hat keine Einschätzung vorgenommen, was dafür spricht, die Ergebnisse als bedeutsam zu interpretieren und die Medienkritik der Rezipient*innen ernst zu nehmen. Denn diese Wahrnehmungen und Einschätzungen leiten deren Handeln beispielsweise ihre zukünftige Mediennutzung. Warum sollten sie Nachrichten in etablierten Medien nutzen, wenn sie die Medien dafür nicht als hilfreich erachten? Erfüllen die Medien aus der individuellen Sicht die normativen Leistungen nicht, so wird auch das Medienvertrauen sinken, möglicherweise bis hin zu Medienzynismus.

Alles in allem stellt dieser empirische Blick auf die Performanz der Medien aus Publikumssicht eine relevante Erweiterung der bisherigen Forschung dar, der aufzeigt, in welchen Bereichen Rezipient*innen unzufrieden mit den gesamtgesellschaftlichen Leistungen der Medien sind.

Förderung Das Projekt wurde durch das Zukunftskonzept LMUexcellent im Rahmen der Exzellenzinitiative gefördert.

Funding Open Access funding provided by Projekt DEAL.

Open Access Dieser Artikel wird unter der Creative Commons Namensnennung 4.0 International Lizenz veröffentlicht, welche die Nutzung, Vervielfältigung, Bearbeitung, Verbreitung und Wiedergabe in jeglichem Medium und Format erlaubt, sofern Sie den/die ursprünglichen Autor(en) und die Quelle ordnungsgemäß nennen, einen Link zur Creative Commons Lizenz beifügen und angeben, ob Änderungen vorgenommen wurden.

Die in diesem Artikel enthaltenen Bilder und sonstiges Drittmaterial unterliegen ebenfalls der genannten Creative Commons Lizenz, sofern sich aus der Abbildungslegende nichts anderes ergibt. Sofern das betreffende Material nicht unter der genannten Creative Commons Lizenz steht und die betreffende Handlung nicht nach gesetzlichen Vorschriften erlaubt ist, ist für die oben aufgeführten Weiterverwendungen des Materials die Einwilligung des jeweiligen Rechteinhabers einzuholen.

Weitere Details zur Lizenz entnehmen Sie bitte der Lizenzinformation auf http://creativecommons.org/ licenses/by/4.0/deed.de.

\section{Literatur}

Arceneaux, K., Johnson, M., \& Murphy, C. (2012). Polarized political communication, oppositional media hostility, and selective exposure. Journal of Politics, 74, 174-186.

Arnold, K. (2009). Qualitätsjournalismus. Die Zeitung und ihr Publikum. Konstanz: UVK.

Arnold, K., \& Wagner, A.-L. (2018). Die Leistungen des Lokaljournalismus. Publizistik, 63, 177-206.

Ball-Rokeach, S. J., \& DeFleur, M. L. (1976). A dependency model of mass-media effects. Communication Research, 3, 3-21. 
Barnidge, M., \& Rojas, H. (2014). Hostile media perceptions, presumed media influence, and political talk. Expanding the corrective action hypothesis. International Journal of Public Opinion Research, 26, 135-156.

Beaudoin, C.E. (2008). Explaining the relationship between internet use and interpersonal trust. Taking into account motivation and information overload. Journal of Computer-Mediated Communication, $13,550-568$.

Bennett, S.E., Rhine, S.L., \& Flickinger, R.S. (2001). Assessing Americans' opinions about the news media's fairness in 1996 and 1998. Political Communication, 18(2), 163-182.

Blöbaum, B. (Hrsg.). (2016). Trust and communication in a digitized world: models and concepts of trust research. Cham: Springer.

BR.de (2016). Medien in der Glaubwürdigkeitskrise? http://www.br.de/presse/inhalt/pressemitteilungen/ glaubwuerdigkeitsstudie-br-b5-geburtstag-100.html. Zugegriffen: 26. Juni 2017.

British Broadcasting Corporation (2004). Building public value. Renewing the BBC for a digital world. London: BBC.

Burkart, R. (2019). Kommunikationswissenschaft. Grundlagen und Problemfelder. Umrisse einer interdisziplinären Sozialwissenschaft (5. Aufl.). Wien: UTB.

Cohen, J., Tsfati, Y., \& Sheafer, T. (2008). The influence of presumed media influence in politics: Do politicians' perceptions of media power matter? Public Opinion Quarterly, 72, 331-344.

Donsbach, W., Rentsch, M., Schielicke, A. M., \& Degen, S. (2009). Entzauberung eines Berufs. Was die Deutschen vom Journalismus erwarten und wie sie enttäuscht werden. Konstanz: UVK.

Fawzi, N., \& Fortkord, C. (2017). Do the media fulfil their functions? The mass media's political and social functions from a recipients' perspective. In 67. Jahreskonferenz der International Communication Association. San Diego, CA, USA.

Fawzi, N., \& Obermaier, M. (2019). Unzufriedenheit - Misstrauen - Ablehnung journalistischer Medien. Eine theoretische Annäherung an das Konstrukt Medienverdrossenheit. Medien und Kommunikationswissenschaft, 67, 27-44.

Gehrau, V. (2008). Fernsehbewertung und Fernsehhandlung. Ansätze und Daten zu Erhebung, Modellierung und Folgen von Qualitätsurteilen des Publikums über Fernsehangebote. München: Reinhard Fischer.

Gil de Zúñiga, H., \& Hinsley, A. (2013). The press versus the public. Journalism Studies, 14, 926-942.

Görke, A. (2014). Vom Hasen und vom Igel - oder warum der Journalismus sein Publikum stets erfolgreich und folgenreich verfehlen darf. In W. Loosen \& M. Dohle (Hrsg.), Journalismus und (sein) Publikum. Schnittstellen zwischen Journalismusforschung und Rezeptions- und Wirkungsforschung (S. 35-51). Wiesbaden: Springer VS.

Graber, D. (2003). The media and democracy. Beyond myths and stereotypes. Annual Review of Political Science, 6, 139-160.

Gunther, A., \& Liebhart, J. L. (2005). Broad reach or biased source? Decomposing the hostile media effect. Journal of Communication, 56, 449-466.

Hagen, L.M. (1995). Informationsqualität von Nachrichten: Meßmethoden und ihre Anwendung auf die Dienste von Nachrichtenagenturen. Opladen: Westdeutscher Verlag.

Hanitzsch, T. (2011). Populist disseminators, detached watchdogs, critical change agents and opportunist facilitators. Professional milieus, the journalistic field and autonomy in 18 countries. International Communication Gazette, 73, 477-494.

Hanitzsch, T., van Dalen, A., \& Steindl, N. (2018). Caught in the nexus. A comparative and longitudinal analysis of public trust in the press. International Journal of Press/Politics, 23(1), 3-23.

Hasebrink, U. (2011). Giving the audience a voice: the role of research in making media regulation more responsive to the needs of the audience. Journal of Information Policy, 1, 321-336.

Heider, D., McCombs, M., \& Poindexter, P. M. (2005). What the public expects of local news. Views on public and traditional journalism. Journalism \& Mass Communication Quarterly, 82, 952-967.

Herzog, A., Hasebrink, U., \& Eilders, C. (2006). Medien-Qualitäten aus der Sicht des Publikums. Europas Mediennutzer zwischen Konsum, Kritik und Partizipation. In S. Weischenberg, W. Loosen \& M. Beuthner (Hrsg.), Medien-Qualitäten. Öffentliche Kommunikation zwischen ökonomischem Kalkül und Sozialverantwortung (S. 399-414). Konstanz: UVK.

Jackob, N. (2010). No alternatives? The relationship between perceived media dependency, use of alternative information sources, and general trust in mass media. International Journal of Communication, 4, 589-606.

Jackob, N. (2012). Gesehen, gelesen - geglaubt? Warum die Medien nicht die Wirklichkeit abbilden und die Menschen ihnen dennoch vertrauen. München: Olzog. 
Jandura, O., \& Kösters, R. (2018). Mediale Repräsentationslücken. In C. Meißelbach, J. Lempp \& S. Dreischer (Hrsg.), Politikwissenschaft als Beruf(S. 111-118). Wiesbaden: Springer.

Johnson, T. J., \& Kaye, B. K. (1998). Cruising is believing? Comparing internet and traditional sources on media credibility measures. Journalism \& Mass Communication Quarterly, 75, 325-340.

Kiousis, S. (2001). Public trust or mistrust? Perceptions of media credibility in the information age. Mass Communication und Society, 4, 381-403.

Kohring, M. (2004). Vertrauen in Journalismus. Theorie und Empirie. Konstanz: UVK.

Kohring, M., \& Matthes, J. (2004). Revision und Validierung einer Skala zur Erfassung von Vertrauen in Journalismus. Medien und Kommunikationswissenschaft, 52, 377-385.

Ladd, J.M. (2012). Why Americans hate the media and how it matters. Princeton: Princeton University Press.

Lieske, S. (2008). Das Image von Journalisten. Eine qualitative Untersuchung. Wiesbaden: VS.

Loosen, W., Schmidt, J.-H., Heise, N. und Reimer, J. (2013). Publikumsinklusion bei einem ARD-Polittalk. Zusammenfassender Fallstudienbericht aus dem DFG-Projekt „Die (Wieder-)Entdeckung des Publikums “. Hamburg: Hans-Bredow-Institut.

Martens, H., \& Hobbs, R. (2015). How media literacy supports civic engagement in a digital age. Atlantic Journal of Communication, 23(2), 120-137.

Martinsen, R. (2009). Öffentlichkeit in der „Mediendemokratie“ aus der Perspektive konkurrierender Demokratietheorien. In F. Marcinkowski \& B. Pfetsch (Hrsg.), Politik in der Mediendemokratie (S. 37-69). Wiesbaden: VS.

McQuail, D. (1992). Media performance: mass communication and the public interest. London: SAGE.

Müller, J. (2013). Mechanisms of trust. News media in democratic and authoritarian regimes. Frankfurt a.M.: Campus.

Neuberger, C. (1997). Was das Publikum wollen könnte. In H. Weßler, C. Matzen, O. Jarren \& U. Hasebrink (Hrsg.), Perspektiven der Medienkritik: Die gesellschaftliche Auseinandersetzung mit öffentlicher Kommunikation in der Mediengesellschaft. Dieter Roß zum 60. Geburtstag (S. 171-184). Opladen: Westdeutscher Verlag.

Neuberger, C. (2004). Qualität im Onlinejournalismus. In K. Beck, W. Schweiger \& W. Wirth (Hrsg.), Gute Seiten - schlechte Seiten. Qualität in der Onlinekommunikation (S. 32-57). München: Reinhard Fischer.

Neuberger, C. (2011). Definition und Messung publizistischer Qualität im Internet: Herausforderungen des Drei-Stufen-Tests. Berlin: Vistas.

Neuberger, C. (2014). Die Identität und Qualität des Journalismus im Internet aus der Sicht des Publikums. In W. Loosen \& M. Dohle (Hrsg.), Journalismus und (sein) Publikum (S. 229-251). Wiesbaden: Springer VS.

Neuberger, C. (2018). Was erwartet die Gesellschaft vom Internet - und was erhält sie? Berlin: KonradAdenauer-Stiftung.

Newman, N., Fletcher, R., Kalogeropoulos, A., \& Nielsen, R. K. (Hrsg.). (2019). Reuters Institute digital news report 2019. Oxford: Reuters Institute for the Study of Journalism.

Norris, P. (2000). A virtuous circle: political communications in postindustrial societies. Cambridge, MA: Cambridge University Press.

Otto, K., \& Köhler, A. (Hrsg.). (2018). Trust in media and journalism. Empirical perspectives on ethics, norms, impacts and populism in Europe. Wiesbaden: Springer VS.

Peifer, J.T. (2018). Perceived news media importance: developing and validating a measure for personal valuations of normative journalistic functions. Communication Methods and Measures, 12(1), 55-79.

Prochazka, F., \& Schweiger, W. (2016). Medienkritik online: Was kommentierende Nutzer am Journalismus kritisieren. SCM Studies in Communicationl Media, 5, 454-469.

Pürer, H. (2014). Publizistik- und Kommunikationswissenschaft. Konstanz: UVK.

Reinemann, C., Fawzi, N., \& Obermaier, M. (2017). Die „Vertrauenskrise“ der Medien - Fakt oder Fiktion? Zu Entwicklung, Stand und Ursachen des Medienvertrauens in Deutschland. In V. Lilienthal \& I. Neverla (Hrsg.), „Lügenpresse“. Anatomie eines politischen Kampfbegriffs (S. 77-94). Köln: Kiepenheuer und Witsch.

Rogers, E.M., \& Agarwala-Rogers, R. (1975). Communication in organizations. New York: The Free Press.

Ronneberger, F. (1974). Die politischen Funktionen der Massenmedien. In W. R. Langenbucher (Hrsg.), Zur Theorie der politischen Kommunikation (S. 193-205). München: Piper.

Saxer, U. (1974). Funktionen der Massenmedien in der modernen Gesellschaft. In R. Kurzrock (Hrsg.), Medienforschung (S. 22-33). Berlin: Colloquium. 
Schatz, H., \& Schulz, W. (1992). Qualität von Fernsehprogrammen. Kriterien und Methoden zur Beurteilung von Programmqualität im dualen Fernsehsystem. Media Perspektiven, o. J.(11), 690-712.

Schielicke, A. M., Mothes, C., \& Donsbach, W. (2014). Vertrauen in Journalismus. Trends und Einflussfaktoren. In B. Stark, O. Quiring \& N. Jackob (Hrsg.), Von der Gutenberg-Galaxis zur Google-Galaxis. Alte und neue Grenzvermessungen nach 50 Jahren DGPuK (S. 247-269). Konstanz: UVK.

Scholl, A., Malik, M., \& Gehrau, V. (2014). Journalistisches Publikumsbild und Publikumserwartungen. Eine Analyse des Zusammenhangs von journalistischen Vorstellungen über das Publikum und Erwartungen des Publikums an den Journalismus. In W. Loosen \& M. Dohle (Hrsg.), Journalismus und (sein) Publikum. Schnittstellen zwischen Journalismusforschung und Rezeptions- und Wirkungsforschung (S. 17-33). Wiesbaden: Springer VS.

Schröter, C. (2016). Themenwoche „Heimat“: Angebot, Rezeption und Urteile des Publikums. Media Perspektiven, o. J.(12), 598-606.

Schudson, M. (2008). Why democracies need an unlovable press. Malden: Polity Press.

Schultz, T., Jackob, N., Ziegele, M., Quiring, O., \& Schemer, C. (2017). Erosion des Vertrauens zwischen Medien und Publikum? Ergebnisse einer repräsentativen Bevölkerungsumfrage. Media Perspektiven, o. J.(5), 246-259.

Schweiger, W. (2017). Der (des)informierte Bürger im Netz. Wie soziale Medien die Meinungsbildung verändern. Wiesbaden: Springer.

Serong, J. (2015). Medienqualität und Publikum. Zur Entwicklung einer integrativen Qualitätsforschung. Konstanz: UVK.

Strömbäck, J. (2005). In search of a standard: Four models of democracy and their normative implications for journalism. Journalism Studies, 6(3), 331-345.

Tsfati, Y., \& Cappella, J. N. (2003). Do people watch what they do not trust? Exploring the association between news media skepticism and exposure. Communication Research, 30, 504-529.

Tsfati, Y., \& Cohen, J. (2005). Democratic consequences of hostile media perceptions. The case of Gaza settlers. Harvard International Journal of Press/Politics, 10(4), 28-51.

Tsfati, Y., Meyers, O., \& Peri, Y. (2006). What is good journalism? Comparing Israeli public and journalists' perspectives. Journalism, 7(2), 152-173.

Vallone, R.P., Ross, L., \& Lepper, M.R. (1985). The hostile media phenomenon. Biased perception and perceptions of media bias in coverage of the Beirut massacre. Journal of Personality und Social Psychology, 49, 577-585.

Van der Wurff, R., \& Schönbach, K. (2014). Civic and citizen demands of news media and journalists. What does the audience expect from good journalism? Journalism \& Mass Communication Quarterly, 91(3), 433-451.

Van Eimeren, B., Simon, E., \& Riedl, A. (2017). Medienvertrauen und Informationsverhalten von politischen Zweiflern und Entfremdeten. Media Perspektiven, o. J.(11), 538-554.

Voigt, J. (2016). Nachrichtenqualität aus Sicht der Mediennutzer. Wie Rezipienten die Leistung des Journalismus beurteilen können. Wiesbaden: Springer VS.

Voltmer, K. (1999). Medienqualität und Demokratie. Eine empirische Analyse publizistischer Informations- und Orientierungsleistungen in der Wahlkampfkommunikation. Baden-Baden: Nomos.

Vowe, G., \& Wolling, J. (2004). Radioqualität - was die Hörer wollen und was die Sender bieten. Vergleichende Untersuchung zu Qualitätsmerkmalen und Qualitätsbewertungen von Radioprogrammen in Thüringen, Sachsen-Anhalt und Hessen. München: kopaed.

Weischenberg, S., Malik, M., \& Scholl, A. (2006). Die Souffleure der Mediengesellschaft. Report über die Journalisten in Deutschland. Konstanz: UVK.

Wellbrock, C.-M., \& Klein, K. (2014). Journalistische Qualität - eine empirische Untersuchung des Konstrukts mithilfe der Concept Map Methode. Publizistik, 59, 387-410.

Wolling, J. (2002). Aufmerksamkeit durch Qualität? Empirische Befunde zum Verhältnis von Nachrichtenqualität und Nachrichtenutzung. In A. Baum \& S. J. Schmidt (Hrsg.), Fakten und Fiktionen: über den Umgang mit Medienwirklichkeiten (S. 202-216). Konstanz: UVK.

Wolling, J. (2004). Qualitätserwartungen, Qualitätswahrnehmungen und die Nutzung von Fernsehserien. Publizistik, 49, 171-193.

Ziegele, M., Schultz, T., Jackob, N., Granow, V., Quiring, O., \& Schemer, C. (2018). Mainzer Langzeitstudie „Medienvertrauen“: Lügenpresse-Hysterie ebbt ab. Media Perspektiven, o. J.(4), 150-161.

Dr. Nayla Fawzi ist akademische Rätin a. Z. am Institut für Kommunikationswissenschaft und Medienforschung der LMU München. 